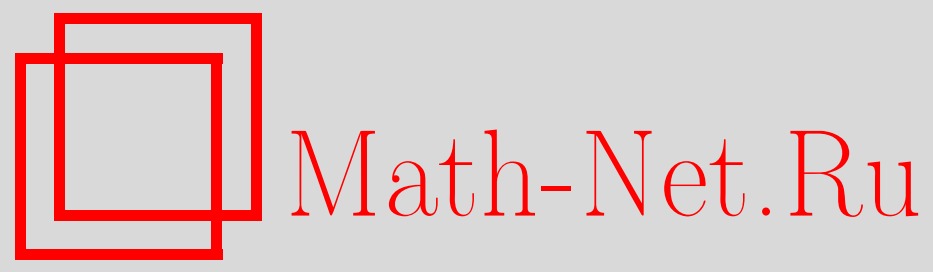

М. И. Тихомирова, В. П. Чистяков, Некоторые характеристики двухэтапных планов экспериментов, Дискрет. матем., 1999, том 11, выпуск 3, 83-90

DOI: https://doi.org/10.4213/dm378

Использование Общероссийского математического портала Math-Net.Ru подразумевает, что вы прочитали и согласны с пользовательским соглашением http: //www . mathnet.ru/rus/agreement

Параметры загрузки:

IP : 44.207 .124 .84

26 апреля 2023 г., 16:29:02

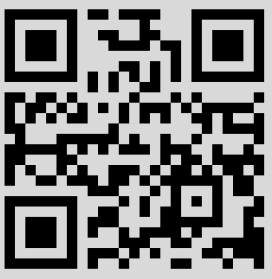




\title{
Некоторые характеристики двухэтапных планов экспериментов
}

\author{
(C) 1999 г. .И. Тихомирова, В. П. Чистяков
}

Процедура выбора объекта с особыми свойствами нередко проводится в два этапа. Сначала по упрощенным измерениям выбирают подозрительные объекты, а затем их исследуют более детально, начиная с самых подозрительных. Важной характеристикой такой процедуры является величина $\tau$, равная числу детально обследованных объектов на втором этапе. В данной работе исследуется распределение величины $\tau$. Результаты применяются к оценке качества двухэтапной процедуры выбора из нескольких полиномиальных гипотез.

Работа выполнена при поддержке Российского фонда фундаментальных исследований, проект 96-15-96092.

\section{1. Введение}

Математическая постановка задачи о наилучшем выборе плана эксперимента и метода обработки наблюдений в общем случае затруднительна. Математическая задача более успешно ставится и решается для достаточно узких множеств рассматриваемых планов. Наиболее полно изучен классический случай, когда по выборке заданного объема требуется выбрать одну из двух гипотез (см. [1]). Качество статистического критерия для многих приложений в этом случае достаточно характеризовать ошибками первого и второго родов. При последовательном наборе наблюдений (см. [2]) для характеристики всей процедуры принятия решения к ошибкам первого и второго родов добавляется математическое ожидание числа наблюдений.

В качестве статистик критериев нередко используются порядковые статистики (члены вариационного ряда), построеннве по самой выборке или по некоторым функциям от нее. Достаточно полно изучены порядковые статистики, построенные по независимым и одинаково распределенным случайным величинам. В книгах $[3,4]$ приводятся распределения порядковых статистик, изучено их асимптотическое поведение, указываются различные приложения.

Многими авторами изучались порядковые статистики, построенные по $s$-цепочкам $(s \geqslant 1)$ исходов полиномиальной схемы или цепи Маркова для большого числа исходов и испытаний (см. $[5-7,13])$. В этих работах рассматривались распределения порядковых статистик.

Необходимость изучения других характеристик вариационного ряда возникает в задачах, когда по наблюдениям некоторых объектов (партии изделий, проб радиоактивного грунта и т. п.) нужно отобрать объекты с особыми свойствами. Нередко эта 
процедура проводится в два этапа. Сначала по упрощенным измерениям выбирают подозрительные объекты, а затем исследуют их более детально, начиная с самых подозрительных.

Такого рода процедуры широко используются в медицинской практике (см., например, [8], стр. 349). Для выявления редкого заболевания среди большой группы доноров делят эту группу на несколько частей и проводят анализ смешанной крови для каждой части, затем в части с обнаруженным заболеванием проводят уже индивидуальный анализ. При поиске полезных ископаемых можно сначала собрать информацию по легко измеряемым признакам, а затем подозрительные участки обследовать более детально (большее количество проб, бурение и т.д.). Во всех подобных случаях стоимость детальных исследований существенно выше предварительных, и потому для оценки процедуры пролезно знать распределение моментов обнаружения разыскиваемых объектов.

В связи с такого рода задачами представляет интерес следующая математическая задача. Пусть по случайным величинам

$$
X_{0}, X_{1}, \ldots, X_{r}
$$

построен вариационный ряд

$$
Z_{(1)}, X_{(2)}, \ldots, Z_{(z+1)} \text {. }
$$

Будем предполагать, что совместная функция распределения величин (1) непрерывна. Требуется исследовать место расположения $\tau$ величины $X_{0}$ в вариационном ряду:

$$
Z_{(r+2-\tau)}=X_{0}
$$

При $\tau=1$ величина $X_{0}$ является максимальным членом вариационного ряда (2), при $\tau=2$ вторым по величине и т. д.

В параграфах 2 и 3 данной работы исследуется распределение величины $\tau$, а в четвертом параграфе полученные результаты применяются к оценке двухэтапной процедуры выбора из нескольких гипотез.

Величины, аналогичные $\tau$ возникают также при исследовании эффективности методов оценки параметров, принимающих значения из конечного множества (см., например, [14]).

\section{2. Зависимые статистики}

Для моментов $\mathbf{M} \tau^{[k]}, \tau^{[k]}=\tau(\tau-1) \ldots(\tau-k+1)$, и вероятностей $\mathbf{P}\{\tau=k\}$ нетрудно получить формулы, которые могут быть использованы при численных расчетах. Приведем соответствующее утверждение в виде следующей теоремы.

Теорема 1. При любой целом $r \geqslant 1$

$$
\begin{aligned}
& \mathbf{M}(\tau-1)^{[k]}=k ! S_{k}, \\
& 1 \leqslant k \leqslant r, \\
& \mathbf{P}\{\tau=k+1\}=\sum_{l=0}^{r-k}(-1)^{l} S_{k+l}\left(\begin{array}{c}
k+l \\
k
\end{array}\right), \quad 0 \leqslant k \leqslant r \\
& \mathbf{P}\{\tau \geqslant k+1\}=\sum_{l=0}^{r-k} S_{k+l}\left(\begin{array}{c}
k+l-1 \\
k-1
\end{array}\right), \quad 1 \leqslant k \leqslant r
\end{aligned}
$$


¿de

$$
\begin{aligned}
& S_{0}=1, \quad U_{i}=\left\{X_{i}>X_{0}\right\}, \quad i=1, \ldots, r \\
& S_{v}=\sum_{1 \leqslant i_{1}<\ldots, i_{v} \leqslant r} \mathbf{P}\left\{U_{i_{1}} \ldots U_{i_{v}}\right\}, \quad v \geqslant 1 .
\end{aligned}
$$

Доказателъство. Нетрудно видеть, что

$$
\tau=1+\chi\left(U_{1}\right)+\ldots+\chi\left(U_{r}\right)
$$

и

$$
(\tau-1)^{[k]}=(\tau-1) \ldots(\tau-k)=\sum_{\left(i_{1}, \ldots, i_{k}\right) \in \mathscr{M}} \chi\left(U_{i_{1}}\right) \ldots \chi\left(U_{i_{k}}\right),
$$

где $\chi(A)$ - индикатор события и

$$
\mathscr{M}=\left\{\left(i_{1}, \ldots, i_{k}\right\}: i_{l}=1, \ldots, r, l=1, \ldots, k ; i_{l_{1}} \neq i_{l_{2}} \text { при любых } l_{1} \neq l_{2}\right\} .
$$

Равенство (4) следует из (7) и (9). Событие $\{\tau-1=k\}$ равносильно осуществлению ровно $k$ событий $U_{1}, \ldots, U_{r}$ и, следовательно, (5) является переформулировкрй результатов из книги Феллера ([9], стр. 124-125). Аналогично получается формула (6) из формулы (5.2) на стр. 128 книги [9]. Теорема доказана.

Из формул (4) легко получить, что

$$
\mathbf{M} \tau=1+S_{1}, \quad \mathbf{D} \tau=S_{2}+S_{1}-S_{1}^{2},
$$

где

$$
S_{1}=\sum_{i=1}^{r} \mathbf{P}\left\{X_{i}>X_{0}\right\}, \quad S_{2}=\sum_{1 \leqslant i<j \leqslant r} \mathbf{P}\left\{X_{i}>X_{0}, X_{j}>X_{0}\right\} .
$$

Многие задачи приводят к необходимости исследовать $\tau$, когда случайные величины (1) имеют совместное нормальное распределение. В этом случае для величин $S_{k}$ можно написать более явное выражение.

Пусть величины (1) имеют невырожденное нормальное распределение с математическими ожиданиями $\mathbf{M} X_{i}=A_{i}$, дисперсиями $\mathbf{D} X_{i}=B_{i}^{2}$ и ковариациями $\operatorname{cov}\left(X_{i} X_{j}\right)=R_{i j} B_{i} B_{j}, i \neq j, i, j=0,1, \ldots, r$. События $U_{i}$ можно записать в виде $U_{i}=\left\{Y_{i}>0\right\}$, где $Y_{i}=X_{i}-X_{0}, i=1, \ldots, r$. Величины $Y_{1}, \ldots, Y_{r}$ имеют совместное нормальное распределение с параметрами

$$
\begin{gathered}
a_{i}=\mathbf{M} Y_{i}=A_{i}-A_{0}, \quad \sigma^{2}=\mathbf{D} Y_{i}=B_{i}^{2}+B_{0}^{2}-2 R_{0 i} B_{0} B_{i}, \\
\operatorname{cov}\left(Y_{i} Y_{j}\right)=\rho_{i j} \sigma_{i} \sigma_{j}, \quad i, j=1, \ldots, r,
\end{gathered}
$$

где

$$
\rho_{i j}=\frac{1}{\sigma_{i} \sigma_{j}}\left(R_{i j} B_{i} B_{j}-R_{0 j} B_{0} B_{j}-R_{0 i} B_{0} B_{i}+B_{0}^{2}\right) .
$$

Вероятность события $U_{i_{1}} \ldots U_{i_{v}}$ находится по формуле

$$
\mathbf{P}\left(U_{i_{1}} \ldots U_{i_{v}}\right)=\int_{\Delta_{i_{1}}}^{\infty} \ldots \int_{\Delta i_{v}} p_{i_{1} \ldots i_{v}}\left(u_{1}, \ldots, u_{v}\right) d u_{1} \ldots d u_{v}
$$


где $\Delta_{i}=a_{i} / \sigma_{i}, p_{i_{1} \ldots i_{v}}\left(u_{1}, \ldots, u_{v}\right)$ - совместная плотность нормального распределения с нулевым математическим ожиданием и ковариационной матрицей $\left(\rho_{i_{k} i_{l}}\right)_{k, l=1}^{v}$, $\rho_{i_{k} i_{k}}=1$.

При $v=2$ для вычисления (13) можно воспользоваться разложением плотности (см. [1], стр. 320) в ряд по степеням $\rho_{i_{1} i_{2}}$. Многочисленные обобщения этого разложения (см., например, $[10,11])$ можно использовать при $v>2$.

\section{3. Независимые статистики}

Пусть статистики (1) независимы и функции распределения

$$
G(x)=\mathbf{P}\left\{X_{0}<x\right\}, \quad F_{i}(x)=\left\{\left\{X_{i}<x\right\}, \quad i=1, \ldots, r\right.
$$

непрерывны. Распределение величинв $\tau$ в рассматриваемом случае удобно искать в виде

$$
\mathbf{P}\{\tau \geqslant k+1\}=\mathbf{M}\left(\mathbf{P}\left\{\eta_{r} \geqslant k \mid X_{0}\right\}\right),
$$

где $\eta_{r}=\chi\left(U_{1}\right)+\ldots+\chi\left(U_{r}\right)$.

Отметим, что условное математическое ожидание

$$
M_{r}(x)=\mathbf{M}\left(\chi\left(U_{1}\right)+\ldots+\chi\left(U_{r}\right) \mid X_{0}=x\right)
$$

определяется формулой

$$
M_{r}(x)=\sum_{l=1}^{r}\left(1-F_{l}(x)\right) .
$$

Обозначим $u_{\gamma}(r)$ корень уравнения

$$
\gamma=\frac{1}{r} M_{r}(x)
$$

Теорема 2. Если при $r \rightarrow \infty$ корень $u_{\gamma}(r)$ уравнения (16) сходится $\kappa u_{\gamma}$, то при $r \rightarrow \infty$

$$
\mathbf{P}\{\tau / r>\gamma\} \rightarrow G\left(u_{\gamma}\right), \quad 0<\gamma<1 .
$$

Доказателъство. Вероятность $\mathbf{P}\{\tau / r>\gamma\}=\mathbf{P}\{\tau>[r \gamma]+1\}$, используя (14), представим в виде

$$
\mathbf{P}\{\tau>\gamma r\}=\int_{-\infty}^{\infty} \mathbf{P}\left\{\frac{1}{r}\left(\eta_{r}-M_{r}(x)\right) \geqslant \frac{1}{r}[\gamma r]-\frac{1}{r} M_{r}(x) \mid X_{0}=x\right\} d G(x) .
$$

При условии $X_{0}=x$ индикаторы $\chi\left(U_{i}\right), i=1, \ldots, r$, независимы и

$$
\mathbf{P}\left\{\chi\left(U_{i}\right)=1 \mid X_{0}=x\right\}=1-F_{i}(x), \quad i=1, \ldots, r .
$$

По закону больших чисел последовательность $\left(\eta_{r}-M_{r}(x)\right) / r$ сходится к нулю по вероятности. Таким образом, если $x$ фиксировано и $r \rightarrow \infty$, то

$$
\mathbf{P}\left\{\frac{1}{r}\left(\eta_{r}-M_{r}(x)\right) \geqslant \frac{1}{r}[\gamma r]-\frac{1}{r} M_{r}(x) \mid X_{0}=x\right\} \rightarrow \begin{cases}1 & \text { при } x<u_{\gamma}, \\ 0 & \text { при } x>u_{\gamma},\end{cases}
$$


так как неравенство $\gamma-M_{r}(x) / r<0$ выполняется при $x<u_{\gamma}(r)$, а по условию теоремы $u_{\gamma}(r) \rightarrow u_{\gamma}$. Переходя в $(17)$ к пределу под знаком интеграла при $r \rightarrow \infty$, получим, что

$$
\lim _{r \rightarrow \infty} \mathbf{P}\{\tau>\gamma r\}=\int_{-\infty}^{u_{\gamma}} d G(x)=G\left(u_{\gamma}\right)
$$

Теорема доказана.

Отметим, что утверждение теоремы сохранится при любых условиях на случайные величины (1), обеспечивающих выполнение (18).

Следствие 1. Если $F_{i}(x)=F(x), i=1, \ldots, r$, mo прu $r \rightarrow \infty$

$$
\mathbf{P}\{\tau / r>\gamma\} \rightarrow G\left(u_{\gamma}\right)
$$

где $u_{\gamma}$ - квантиль распределения $F(x)$, то есть $1-F\left(u_{\gamma}\right)=\gamma$.

Если $G(x)=F(x)$, то $G\left(u_{\gamma}\right)=1-\gamma$, и предельным распределением для $\tau / r$ оказывается равномерное распределение на $[0,1]$. Последнее утверждение легко следует из очевидного в этом случае равенства

$$
\mathbf{P}\left\{X_{i_{1}}<\ldots<X_{i_{r+1}}\right\}=\frac{1}{(r+1) !}
$$

при любой перестановке $\left(i_{1}, \ldots, i_{r+1}\right)$ чисел $(0,1, \ldots, r)$.

Отметим, что в условиях следствия 1 упрощаются формулы (5) и (6). Например, вероятность $\mathbf{P}\{\tau=k+1\}$ можно записать в виде

$$
\mathbf{P}\{\tau=k+1\}=\int_{-\infty}^{\infty}\left(\begin{array}{l}
r \\
k
\end{array}\right)(1-F(x))^{k} f^{r-k}(x) d G(x) .
$$

Эту формулу легко получить непосредственно. Нужно воспользоваться тем, что величина $\eta_{r}=\chi\left(U_{1}\right)+\ldots+\chi\left(U_{r}\right)$ при условии $X_{0}=x$ является числом успехов в схеме Бернулли с $r$ испытаниями и вероятностью успеха $1-F(x)$.

\section{4. Выборы из нескольких гипотез}

Независимые случайные величины

$$
x_{1}, x_{2}, \ldots, x_{n}
$$

распределены в соответствии с одной из $r$ гипотез $H_{1}, \ldots, H_{r}$. При гипотезе $H_{l}$, $l=1, \ldots, r$

$$
\mathbf{P}\left\{x_{t}=k\right\}=p_{l k}>0, \quad k=1, \ldots, N, \quad t=1, \ldots, n .
$$

Будем полагать, что на гипотезах задано априорное распределение вероятностей $p_{1}, \ldots, p_{r}$. Гипотеза $H_{l}$ выбирается с вероятностью $p_{l}, l=1, \ldots, r$. Обозначим $\theta$ номер выбранной гипотезы.

Распределение вероятностей, математическое ожидание, дисперсию и ковариацию при гипотезе $H_{l}$ будем обозначать соответственно $\mathbf{P}_{l}, \mathbf{M}_{l}, \mathbf{D}_{l}, \operatorname{cov}_{l}$. 
По реализации (20) и дополнительным наблюдениям нужно найти гипотезу, по которой была получена данная реализация. Рассмотрим следующую двухэтапную процедуру определения истинной гипотезы.

На первом этапе находится реализация (20), по ней вычисляются частоты исходов $\nu_{1}=\nu_{1}(n), \ldots, \nu_{N}=\nu_{N}(n)$ и статистики

$$
\xi_{i}(n)=\ln p_{i}+\sum_{k=1}^{N} \nu_{k}(n) \ln p_{i k}, \quad i=1, \ldots, r .
$$

Номер наибольшей статистики по байесовскому критерию (см. [12], стр. 273) объявляется номером истинной гипотезы.

По статистикам (22) строится вариационный ряд

$$
\xi_{(1)} \leqslant \xi_{(2)} \leqslant \ldots \leqslant \xi_{(r)}
$$

Если реализации всех статистик различны, то равенства

$$
\xi_{(k)}=\xi_{i_{k}}, \quad k=1, \ldots, r, \quad i_{k} \in\{1,2, \ldots, r\}
$$

однозначно определяют перестановку

$$
\left(i_{1}, i_{2}, \ldots, i_{r}\right)
$$

номеров $(1,2, \ldots, r)$. Если среди реализаций величин $(22)$ оказались равные, например, одинаковыми оказались $s$ значений статистик, то в (23) этим статистикам соответствует $s$ одинаковых членов вариационного ряда, и порядок расположения номеров этой группы не определен. В этом случае, порядок расположения номеров группы будем задавать произвольным образом, например, выбирать расположения равновероятно. Таким образом, последовательность (24) будет полностью определена в любом случае.

На втором этапе проводятся более полные испытания, по которым однозначно решается, является ли истинной гипотеза $H_{i_{r}}$, соответствующая наибольшему члену вариационного ряда. Если окажется, что $\theta=i_{r}$, то решение найдено и испытания прекращаются. Если окажется, что $\theta \neq i_{r}$, то по новой серии дополнительных испытаний аналогично проверяется гипотеза $\theta=i_{r-1}$ и так далее. Такая процедура с вероятностью единица закончится правильным решением.

Существенной характеристикой процедуры является величина $\tau$, определяющая место, на которое попала статистика $\xi_{\theta}: \tau=l$, если $\theta=i_{r+1-l}, l=1, \ldots, r$. При любом способе упорядочивания членов вариационного ряда (23) справедливы неравенства $\underline{\tau} \leqslant \tau \leqslant \bar{\tau}$, где

$$
\begin{aligned}
& \underline{\tau}=1+\sum_{i=1}^{r} \underline{\chi}_{i}, \quad \bar{\tau}=1+\sum_{i=1}^{r} \bar{\chi}_{i}, \\
& \underline{\chi}_{i}=\chi\left\{\xi_{i}>\xi_{\theta}\right\}, \quad \bar{\chi}_{i}=\chi\left\{\xi_{i} \geqslant \xi_{\theta}\right\}, \quad i=1, \ldots, r, \quad i \neq \theta .
\end{aligned}
$$

Здесь и далее в суммы со знаком * не входят слагаемые с индексами, равными $\theta$.

Будем рассматривать асимптотическое поведение $\tau$ при $n \rightarrow \infty$ и фиксированных $r$ и $p_{i}, i=1, \ldots, r$, в схеме серий, когда

$$
p_{i k}=\frac{1}{N}\left(1+\frac{w_{i k}(n)}{\sqrt{n}}\right), \quad w_{i k}(n) \rightarrow w_{i k}, \quad k=1, \ldots, N, \quad i=1, \ldots, r .
$$


При условии $\theta=l$ в схеме серий (25) случайные величины $\eta_{l i}=\xi_{i}-\xi_{l}, i \neq l$, асимптотически нормальны. Для мометнов первого и второго порядков этих величин справедливы формулы

$$
\mathbf{M}_{l} \eta_{l i}=n\left(\tilde{a}_{l i}+\frac{1}{n} \ln \frac{p_{i}}{p_{l}}\right), \quad \operatorname{cov}_{l}\left(\eta_{l i} \eta_{l j}\right)=n \tilde{\sigma}_{l i, l j},
$$

где

$$
\tilde{a}_{l i}=\sum_{k=1}^{N} p_{l k} c_{i k}^{(l)}, \quad \tilde{\sigma}_{l i, l j}=c_{i k}^{(l)} c_{j k}^{(l)}-\tilde{a}_{l i} \tilde{a}_{l j}, \quad c_{i k}^{(l)}=\ln \frac{p_{i k}}{p_{l k}} .
$$

Лемма 1. В схеме серий (25) при условии $\theta=l$ существуют предель

$$
\lim _{n \rightarrow \infty} n \tilde{a}_{l i}=-\frac{1}{2} b_{i i}^{(l)}, \quad \lim _{n \rightarrow \infty} n \tilde{\sigma}_{l i, l j,}=b_{i j}^{(l)}
$$

где

$$
b_{i j}^{(l)}=\frac{1}{N} \sum_{k=1}^{N}\left(w_{i k}-w_{l k}\right)\left(w_{j k}-w_{l k}\right) .
$$

Доказательство. Из формул (25) и (27), используя разложение логарифма, получим, что

$$
c_{i k}^{(l)}=\frac{1}{\sqrt{n}}\left(w_{i k}(n)-w_{l k}(n)\right)-\frac{1}{2 n} w_{i k}^{2}(n)+\frac{1}{2 n} w_{i l}^{2}(n)+O\left(\frac{1}{n^{3 / 2}}\right),
$$

и, следовательно,

$$
\begin{aligned}
\tilde{a}_{l i} & =\frac{1}{N} \sum_{k=1}^{N}\left(1+\frac{1}{\sqrt{n}} w_{l k}(n)\right) c_{i k}^{(l)} \\
& =\frac{1}{2 N n} \sum_{k=1}^{N}\left(w_{i k}(n)-w_{l k}(n)\right)^{2}+O\left(\frac{1}{n^{3 / 2}}\right),
\end{aligned}
$$

так как

$$
\sum_{k=1}^{N} w_{i k}(n)=0
$$

Аналогично доказывается другая формула.

Лемма 2. $Е с л и b_{i i}^{(l)}>0, i=1, \ldots, r$, то в схеме серий (25)

$$
\lim _{n \rightarrow \infty} \mathbf{P}\left\{\underline{\eta}_{l i_{1}}>0, \ldots, \eta_{l i_{v}}>0\right\}=p_{i_{1}, \ldots, i_{v}}^{(l)},
$$

гдe

$$
p_{i_{1}, \ldots, i_{v}}^{(l)}=\mathbf{P}\left\{\zeta_{1}>\Delta_{i_{1}}^{(l)}, \ldots, \zeta_{v}>\Delta_{i_{v}}^{(l)}\right\},
$$

$\left(\zeta_{1}, \ldots, \zeta_{v}\right)$ - нормалъно распределенный вектор с нулевым математическим ожиданием и ковариационной матрицей

$$
\left(b_{i_{k} i_{m}}^{(l)}\right), \quad k, m=1, \ldots, v,
$$

$b_{i j}^{(l)}$ определень равенством $(28), \Delta_{l i}^{(l)}=(1 / 2) \sqrt{b_{i i}^{(l)}}$. 
Утверждение леммы 2 следует из леммы 1 и асимптотической нормальности вектора $\left(\nu_{1}(n), \ldots, \nu_{N}(n)\right)$.

Отметим, что вероятности $\mathbf{P}_{l}\left\{\eta_{l i_{1}}>0, \ldots, \eta_{l i_{v}}>0\right\}, \mathbf{P}_{l}\left\{\eta_{l i_{1}} \geqslant 0, \ldots, \eta_{l i_{v}} \geqslant 0\right\}$, входящие в определение величин $\underline{\tau}$ и $\bar{\tau}$, в пределе не различаются. Отсюда, используя неравенства (24), неравенства

$$
\mathbf{M}_{l}(\underline{\tau}-1)^{[k]} \leqslant \mathbf{M}_{l}(\tau-1)^{[k]} \leqslant \mathbf{M}_{l}(\bar{\tau}-1)^{[k]}, \quad \mathbf{P}\{\underline{\tau}<k\} \leqslant \mathbf{P}\{\tau<k\} \leqslant \mathbf{P}\{\bar{\tau}<k\},
$$

лемму 2 и теорему 1 , получаем следующее утверждение.

Теорема 3. Если $b_{i i}^{(l)}>0, i=1, \ldots, r$, то в схеме серий (25)

$$
\begin{gathered}
\lim _{n \rightarrow \infty} \mathbf{M}_{l}(\tau-1)^{[k]}=k ! S_{k}, \quad \lim _{n \rightarrow \infty} \mathbf{P}_{l}\{\tau=k+1\}=\sum_{v=0}^{r-k-1}(-1)^{v} S_{k+v}^{(l)}\left(\begin{array}{c}
k+v \\
k
\end{array}\right), \\
\lim _{n \rightarrow \infty} \mathbf{P}_{l}\{\tau \geqslant k+1\}=\sum_{v=0}^{r-k-1}(-1)^{v} S_{k+v}^{(l)}\left(\begin{array}{c}
k+v-1 \\
k-1
\end{array}\right),
\end{gathered}
$$

¿de

$$
S_{0}=1, \quad S_{v}^{(l)}=\sum_{1 \leqslant i_{1}<\ldots<i_{v} \leqslant r}^{*} p_{i_{1}, \ldots, i_{v}}^{(l)},
$$

$p_{i_{1}, \ldots, i_{v}}^{(l)}$ 'определены формулами $(28),(29)$.

\section{Список литературы}

1. Крамер Г., Математические методы статистики. Мир, Москва, 1975.

2. Вальд А., Последователъный анализ. Наука, Москва, 1960.

3. Дейвид Г., Порлдковые статистики. Наука, Москва, 1979.

4. Галамбош Я., Асимптопическая теория экстремалъных порядковых статистик. Наука, Москва, 1984.

5. Колчин В. Ф., Севастьянов Б. А., Чистяков В. П., Случайные размещения. Наука, Москва, 1976.

6. Ивченко Г. И., О предельных распределениях порядковых статистик полиномиальной схемы. Теория вероятностей и ее применения (1971) 16, №1, 94-107.

7. Амбросимов А. С., Об асимптотическом поведении максимума в однородной цепи Маркова с большим числом состояний. Теория вероятностей и ее применения (1978) $\mathbf{2 3}$, №2, 438-445.

8. Ермаков С. М., Математическая теория планирования эксперимента. Наука, Моква, 1983.

9. Феллер В., Введение в теорию вероятностей и ее приложения. т. 1, Мир, Москва, 1984.

10. Висков О. В., Несколько замечаний о полиномах Эрмита. Теория вероятностей и ее применения (1991) 36, №3, 566-569.

11. Slepian D., On the symmetrized Kroneker power of a matrix and extensions of Mehker's formula for Hermite polynomials. SIAM J. Math. Anal. (1972) 3, №4, 606-616.

12. Боровков А. А., Математическал статистика. Наука, Москва, 1984.

13. Амбросимов А. С., О состоятельности метода максимального правдоподобия в задаче упорядочения исходов полиномиальной схемы в соответствии с их вероятностями. Дискретная математика (1995) 7, №2, 118-130.

14. Амбросимов А. С., Об эффективности метода максимального правдоподобия определения входного слова полунепрерывного канала без памяти при гауссовском шуме. Обозрение прикладной и промычленной математики (1998) 5, №2, 195-196. 